\title{
Shot Noise of Single-Electron Tunneling in 1D Arrays
}

\author{
K. A. Matsuoka and K. K. Likharev \\ Department of Physics and Astronomy \\ State University of New York \\ Stony Brook, NY 11794-3800
}

\begin{abstract}
We have used numerical modeling and a semi-analytical calculation method to find the low frequency value $S_{I}(0)$ of the spectral density of fluctuations of current through 1D arrays of small tunnel junctions, using the "orthodox theory" of single-electron tunneling. In all three array types studied, at low temperature $\left(k_{B} T \ll e V\right)$, increasing current induces a crossover from the Schottky value $S_{I}(0)=2 e\langle\bar{I}\rangle$ to the "reduced Schottky value" $S_{I}(0)=2 e\langle\bar{I}\rangle / N$ (where $N$ is the array length) at some crossover current $I_{c}$. In uniform arrays over a ground plane, $I_{c}$ is proportional to $\exp (-\lambda N)$, where $\lambda^{-1}$ is the single-electron soliton length. In arrays without a ground plane, $I_{c}$ decreases slowly with both $N$ and $\lambda$. Finally, we have calculated the statistics of $I_{c}$ for ensembles of arrays with random background charges. The standard deviation of $I_{c}$ from the ensemble average $\left\langle I_{c}\right\rangle$ is quite large, typically between 0.5 and 0.7 of $\left\langle I_{c}\right\rangle$, while the dependence of $\left\langle I_{c}\right\rangle$ on $N$ or $\lambda$ is so weak that it is hidden within the random fluctuations of the crossover current.
\end{abstract}

73.40.Gk, 73.40.Rw, 85.40.Hp 


\section{INTRODUCTION}

Single-electron tunneling (for general reviews, see Refs. 1,2) is one of the most active areas of solid state physics research, but it has several key problems that have not yet been addressed in detail. One of these problems is the dilemma of the discreteness/continuity of electric charge transfert. Several single-electron tunneling phenomena can be understood as an interplay between discrete transfer of electric charge via electron tunneling, and continuous transfer in ordinary diffusive conductors. For example, a tunnel junction biased by a dc current may generate single-electron tunneling (SET) oscillations with average frequency

$$
f_{S}=\frac{\langle I\rangle}{e}
$$

This effect may be interpreted as a gradual accumulation of continuous charge on the junction capacitance, followed by the abrupt passage of one electron through the junction, as soon as the accumulated charge has reached a threshold level $3 Q_{t}= \pm e / 2$.

However, if the charge transfer in the external circuit (fixing the current $I$ ) is discrete, SET oscillations do not exist. This can be seen from the following general formula for the SET oscillation linewidth1]

$$
\Gamma_{S}=\left(\frac{\pi}{e}\right)^{2} S_{I}(0) \quad\left(\Gamma_{S} \ll f_{S}\right),
$$

where $S_{I}(f)$ is the spectral density of the bias current fluctuations. For example, if the current is fixed using another tunnel junction, then at low temperatures the fluctuations obey the Schottky formula

$$
S_{I}(0)=2 e\langle I\rangle
$$

and $\Gamma_{S} \geq f_{S}$, that is, SET oscillations are completely smeared by the current fluctuations 1 . On the other hand, in macroscopic diffusive conductors, the current noise may be much lower than the Schottky value, and SET oscillations (and very similar "Bloch" oscillations $⿴$ ) may have a relatively narrow bandwidth - see, e.g., experimental measurements in Ref. 5. 
More generally, discreteness of charge transfer will certainly be one of the central issues facing the emerging nanoelectronics. This is why it is important to formulate the conditions under which the transport of charge through a conductor may be considered as (quasi)continuous, i.e., having discreteness $\delta Q \ll e$. The definition of $\delta Q$ can be most readily introduced in the most interesting case of negligible thermal and quantum fluctuations:

$$
\begin{aligned}
k_{B} T & \ll\left[e\langle V\rangle, e V_{t}\right], \\
G & \ll \frac{e^{2}}{h},
\end{aligned}
$$

where $V_{t}$ is the Coulomb blockade threshold of the conductor and $G$ its effective conductance. However, even in this simplest case, the definition depends essentially on the characteristic time scale $\tau$ of an experiment:

A. If $f_{S} \tau \gg 1$ (i.e. either the time scale $\tau$ is large, or the dc current is high, or both), $\delta Q$ may be defined as follows:

$$
\frac{\delta Q}{e}=\frac{S_{I}(0)}{2 e\langle I\rangle}
$$

In fact, if the charge $Q$ transferred through a system may be presented as a Poissonian series of jumps of fixed height $\delta Q$, then repeating the well-known derivation of the Schottky formula we arrive at Eqn. (6). If the jump height is random as well, Eqn. (6) is still applicable as an estimate of the average jump height.

B. In the opposite limit, when $\tau$ is much shorter than the average spacing between the charge jumps $\left(f_{S}^{-1}\right)$, we are essentially dealing with the Coulomb blockade regime. In this case an adequate definition of $\delta Q$ is as follows:

$$
\delta Q=C_{i n} V_{t}
$$

where $C_{i n}$ is the effective input capacitance of the system of interest. ( $\delta Q$ given by this formula is the fraction of the initial electric charge of the system which cannot relax, due to the Coulomb blockade; for a diffusive conductor $\delta Q \rightarrow 0$, while for a single tunnel junction $\delta Q=e / 2$. 
One of the most interesting systems capable of quasicontinuous charge transfer is the 1D array of small tunnel junctions - see, e.g., the review in Ref. 6. The key property of such an array is that each additional electron inserted into one of its islands creates a series of gradually decreasing polarization charges, and hence may be considered as a "single-electron soliton" with a characteristic size $M$ which may be much larger than one island. As a result, when an electron is drifting along the array, tunneling between the neighboring islands, the effective charge $Q$ transferred through the external electrodes - which is essentially what interests us - changes in jumps of scale $\delta Q \sim e / M$ which may be much smaller than $e$.

This does not mean, however, that the condition $M \gg 1$ is sufficient for the quasicontinuous transfer of charge in the arrays. In fact, the calculation of the Coulomb blockade threshold for arrays with uniform 8 and random 910 background charges have shown that, under definition $\mathrm{B}$, charge transport in uniform arrays with vanishing background charge cannot be considered as continuous $(\delta Q \sim e)$. The reason is that a relatively strong Coulomb blockage results from the pinning of single-electron solitons by the sharp edges of the array. Presently, we know only two cases when charge transport through a uniform array is quasicontinuous $(\delta Q \ll e)$ according to the definition B:

1) An array with arbitrary capacitances, but with the background charge of all the islands equal to $\pm e / 2$. (In this case the Coulomb blockade threshold vanishes, and $\delta Q \rightarrow 0$.)

2) An array with $1 \ll N \ll 2 M$ and random background charges. (In this case 1011 $V_{t} \approx 0.5 e N^{1 / 2} / C$, where $C$ is the tunnel junction capacitance, while $C_{i n}=C / N$, and hence $\delta Q / e \approx 1 / 2 N^{1 / 2} \ll 1$.)

However, even in these cases, the charge transfer is not automatically continuous in the sense of criterion A. If the Coulomb blockade is finite, and the array is driven with dc voltage $V$ slightly above the blockade threshold $V_{t}$, one of the junctions presents a bottleneck to the single-electron soliton drift along the array. As a result, the passage of an electron consists of a long wait at the bottleneck junction, followed by a rapid burst of transitions through the remaining junctions of the array. At $V \rightarrow V_{t}$ the statistics of these bursts is always Poissonian, and the charge transferred by each burst is equal to $e$, so that the shot noise is 
well described by the Schottky formula.

Thus, current noise in 1D arrays presents an important problem. To our knowledge, this problem has previously only been solved 22 for a very particular case of uniform arrays with $M \rightarrow \infty$ (zero stray capacitance) and zero background charge. The objective of this work was to calculate $S_{I}(0)$ (and hence the effective discreteness of charge transfer for long time intervals) for a much broader range of array parameters.

\section{BASIC FORMULAS}

We have considered arrays consisting of $N-1$ small metallic islands connected by $N$ tunnel junctions, and flanked at either end by dc voltage-biased electrodes (Fig. 1). Under the conditions expressed by Eqs. (4), (5), we can ignore the effects of cotunneling and of thermally activated tunneling. Current flow in single-electron arrays may be analyzed in terms of the junction tunneling rates, $\Gamma_{i j}$. According to the orthodox theory 8 , at zero temperature,

$$
\Gamma_{i j}= \begin{cases}\frac{G \Delta W_{i j}}{e^{2}} & \Delta W_{i j}>0, \\ 0 & \Delta W_{i j}<0,\end{cases}
$$

where $\Delta W_{i j}$ is the drop in the free (electrostatic) energy, caused by the tunneling event. The drop in energy due to an electron tunneling from island $i$ to island $j$ can be written

$$
\Delta W_{i j}=e^{2} C_{i j}^{-1}-\frac{e^{2} C_{i i}^{-1}+e^{2} C_{j j}^{-1}}{2}+e\left[\phi_{j}-\phi_{i}\right],
$$

where $\vec{\phi}$ is the vector of the electrostatic potential of the islands before the jump, and the matrix $\boldsymbol{C}^{-\mathbf{1}}$ of inverse capacitances is defined by the following equation:

$$
\phi_{i}=\sum_{j \in i s l} C_{i j}^{-1}\left(q_{j}+\tilde{q}_{j}\right), \quad \tilde{q}_{j} \equiv \sum_{k \in e x t} \tilde{C}_{j k} V_{k}
$$

Here the matrix $\tilde{\boldsymbol{C}}$ represents capacitances between islands and external terminals with potentials $V_{k}$. If these potentials do not change in time, the probability that the system preserves its charge state can be expressed explicitly: 


$$
P(t)=\exp \left(-\Gamma\left(t-t_{o}\right)\right),
$$

where $t_{o}$ is the time of the preceding tunneling event, while $\Gamma$ is the total rate for all possible tunneling events:

$$
\Gamma=\sum_{\{i j\} \in j c t} \Gamma_{i j} .
$$

\section{NOISE COMPUTATION}

The preceding relations were incorporated into our main computational tool, a C++

program called msol that uses a Monte Carlo algorithm目 to simulate the flow of current in dc voltage biased 1D arrays. The basic unit of calculation in mso is the "current run", in which charge flows through the array until a user-specified total charge $Q$ is transferred. To calculate $S_{I}(0)$, mso loops through a user-specified number $N_{r}$ of current runs, each starting with the same initial charge state and ending when the total charge transferred equals $Q$. The same random number generators 3 for time and jump location are used continuously through all loops. To the extent that the random numbers constitute a "good" quasi-random series, the ensemble of current runs represents a statistical ensemble of independent, initially identical systems.

We may calculate $S_{I}(0)$ from the statistical properties of the time $T_{Q}$ taken by each run, as 12:

$$
\frac{S_{I}(0)}{2 e\langle\bar{I}\rangle} \approx \frac{Q}{e} \frac{\operatorname{Var}\left(T_{Q}\right)}{\left\langle T_{Q}\right\rangle^{2}},
$$

where $\bar{I}=Q / T_{Q}$, and the angle brackets and variance refer to our statistical ensemble of current runs. Equation (13) is exact only for infinite $Q$ and $N_{r}$; since the jumps are not completely independent, the accuracy of this formula should be determined experimentally. Figure 2 shows a typical dependence of $S_{I}(0) / 2 e\langle\bar{I}\rangle$ on $Q$ and $N_{r}$. The results for $Q \gtrsim 1000 e$, $N_{r} \gtrsim 1000$ seem to be accurate to within $10 \%$ of the asymptotic value. In this paper, we 
used the parameters $Q=1000 e, N_{r}=1000$ for calculating shot noise in arrays without background charge, and assigned $10 \%$ error bars to these numbers.

For arrays with random background charge, each point was calculated for 50 different realizations of the background charge for each circuit, using the parameters $Q=200 e$ and $N_{r}=200$, to keep the simulation time within reasonable limits. Although these calculations are therefore less accurate, perhaps only to within $20 \%$ of their asymptotic value, this inaccuracy was overshadowed by the overall spread in shot noise values among the different background charge realizations.

Calculation (CPU) times in mso scale as $\sim N^{a} Q N_{r}$, with a slightly larger than 2. A typical calculation with $N=20, Q=1000 e$, and $N_{r}=1000$ takes around 400 seconds of CPU time on an AlphaStation 250 (266 MHz Alpha, Digital Unix 4.0b, Digital cxx) or around 950 seconds on a Linux PC (120 MHz Pentium, RedHat Linux 2.0.30, Gnu c++).

\section{CROSSOVER CURRENT}

Varying the bias voltage across an array, we have calculated the average current and spectral density as functions of applied voltage and have made parametric plots of $S_{I}(0) / 2 e\langle\bar{I}\rangle$ vs. $\langle\bar{I}\rangle$ (Fig. 3). We will refer to these plots as $S-I$ curves.

The most immediate, universal result of our calculations is the crossover of $S_{I}(0) / 2 e\langle\bar{I}\rangle$ from 1 to $1 / N$ with increasing current. This result can be understood as follows. As argued in section [I, $S_{I}(0)$ near threshold is dominated by the Poissonian statistics of tunneling through a single bottleneck junction, and is thus given by the Schottky formula:

$$
\left.\frac{S_{I}(0)}{2 e\langle\bar{I}\rangle}\right|_{I \rightarrow 0} \rightarrow 1 .
$$

At high voltages, however, a large number of charge states becomes available for tunneling through each junction. Though the tunneling rate for each of these states may be affected by the state of neighboring junctions, these effects are averaged out, since the voltage depen-

dence of the rate of tunneling through each junction is linear at $\Delta W_{i j}>0$ (see Eqn. (8)). 
Under these conditions, current noise through each junction is described by the Schottky formula, $S_{I}^{(1)}(0)=2 e\langle\bar{I}\rangle$. Since we may transform current noise into voltage noise by the square of the dynamic resistance (which at $V \rightarrow \infty$ just equals $R$ ), we write the voltage noise of a single junction as

$$
\left.S_{V}^{(1)}(0)\right|_{I \rightarrow \infty}=2 e\langle\bar{I}\rangle R^{2}
$$

The total voltage noise $S_{V}$ is the simple sum of the noise of the individual junctions $S_{V}^{(1)}$, while the total current noise is finally obtained from $S_{V}$ via the total array resistance $N R$ :

$$
\begin{aligned}
\left.S_{V}(0)\right|_{I \rightarrow \infty} & =2 e\langle\bar{I}\rangle R^{2} N, \\
\left.S_{I}(0)\right|_{I \rightarrow \infty} & =\frac{S_{V}(0)}{(N R)^{2}}=\frac{2 e\langle\bar{I}\rangle}{N} .
\end{aligned}
$$

(For the particular case $N=2$ this equation has been discussed in Ref. 14.) Thus the crossover in $S_{I}(0) / 2 e\langle\bar{I}\rangle$ from 1 to $1 / N$ with increasing current could be expected; what was really surprising for us is that in most cases this crossover takes place very close to the Coulomb blockade threshold, where the array $I-V$ curve is still not quite linear, and hence the arguments given above cannot be taken too seriously.

In order to describe the crossover quantitatively, we may define the crossover current $I_{c}$ as a value at which $S_{I}(0) / 2 e\langle\bar{I}\rangle$ is midway between these two limits, on a logarithmic scale:

$$
\frac{S_{I}(0)}{2 e I_{c}} \equiv \frac{1}{\sqrt{N}}
$$

We have written a Perl script called sicurve to automate the extraction of the crossover current $I_{c}$ from the $S-I$ curves. While invoking mso, sicurve continuously adjusts the change in bias voltage between successive points, in an attempt to produce a series of evenly spaced points in the $S_{I}(0) / 2 e\langle\bar{I}\rangle$ vs. $\langle\bar{I}\rangle$ plane (see Fig. [). This is an important practical technique for generating $S-I$ curves on circuits with random background charge, since the relationship between voltage, current, and spectral density can be quite irregular. 


\section{MODEL 1: ARRAYS NEAR GROUND PLANE}

Our first case was the simplest model 8 of a uniform, symmetrically biased array near a ground plane with no background charges (Fig. 1b). The direct capacitance matrix in this model is tridiagonal, and is described by one dimensionless parameter, the ratio $C_{0} / C$, where $C$ is the junction capacitance, and $C_{0}$ is the "stray" capacitance between an island and the ground plane. In this model, the reciprocal length scale $\lambda=1 / M$ of the single-electron soliton is determined as

$$
\lambda=\cosh ^{-1}\left(C_{0} / 2 C-1\right),
$$

and in the most interesting limit of $C_{0} \ll C, M=\sqrt{C / C_{0}} \gg 1$.

Surprisingly, our numerical results (Fig. 1) show that for all values of $\lambda$ and $N$, data for $I_{c}$ fall roughly on a single universal curve, with $I_{c} \propto \exp (-N \lambda / 3)$ for $\lambda \gtrsim 10$. For a fixed product $N \lambda$, there is a relatively weak decrease of $I_{c}$ with increasing $N$.

To understand this unexpected result, we began looking for an analytic expression for current noise for the case when the passage of charge through the array consists of a fixed, repeated sequence of tunneling events. If such a sequence is repeated $n=Q / e$ times, we can write the average total time as a sum of average times for each jump in the fixed sequence:

$$
\left\langle T_{Q}\right\rangle=n \sum_{i=1}^{N}\left\langle\delta t_{i}\right\rangle=\frac{Q}{e} \sum_{i=1}^{N} \Gamma_{i}^{-1},
$$

where $\Gamma$ is the total rate at each stage of the process. Since the time of each jump follows Poissonian statistics,

$$
\operatorname{Var}\left(\delta t_{i}\right)=\Gamma_{i}^{-2},
$$

and since the jump times are independent of one another,

$$
\operatorname{Var}\left(T_{Q}\right)=\frac{Q}{e} \sum_{i=1}^{N} \operatorname{Var}\left(\delta t_{i}\right)=\frac{Q}{e} \sum_{i=1}^{N} \Gamma_{i}^{-2}
$$

we arrive at a simple formula12: 


$$
\frac{S_{I}(0)}{2 e\langle\bar{I}\rangle}=\frac{\sum_{i=1}^{N} \Gamma_{i}^{-2}}{\left(\sum_{i=1}^{N} \Gamma_{i}^{-1}\right)^{2}} .
$$

This formula was obtained earlier12 for a particular case $C_{0}=0$. In particular, it shows that the spectral density can be dominated by bottleneck points, where the rate $\Gamma_{i}$ is much lower than average.

In order to find these bottleneck points for the present case of arrays near a ground plane, let us examine the energy profile created by these arrays for tunneling charges. The potential created by an electron in an array with externals at both ends is 15

$$
\phi_{s}\left(n, n^{\prime}\right)=\frac{e}{C_{e f f}}\left\{e^{-\left|n-n^{\prime}\right| \lambda}-\frac{e^{-n \lambda} \sinh \left[\left(N-n^{\prime}\right) \lambda\right]+e^{-(N-n) \lambda} \sinh (n \lambda)}{\sinh (N \lambda)}\right\},
$$

where $n \in\{0,1 \ldots N\}$ is the position of the electron in the array, $n^{\prime}$ is the measurement position, and $C_{e f f} \equiv \sqrt{C_{0}^{2}+4 C C_{0}}$. For our symmetric bias $\left(V_{2}=-V_{1}=V / 2\right)$, the potential created by the external electrodes is 1 国

$$
\phi_{e}(n)=\frac{V}{2} \frac{\sinh [(N / 2-n) \lambda]}{\sinh (N \lambda / 2)}
$$

Numerical simulations show that in symmetrically biased arrays with $\lambda \gtrsim 1$, the basic tunneling scenario near threshold is the passage of electron-hole pairs. The components of the pair enter at opposite ends of the array, move towards each other, then annihilate near the center (see Table 1). We can write the energy of the electron-hole pair as

$$
W\left(n_{1}, n_{2}\right)=W_{o}\left(n_{1}\right)-e \phi_{e}\left(n_{1}\right)+W_{o}\left(n_{2}\right)+e \phi_{e}\left(n_{2}\right)-e \phi_{s}\left(n_{1}, n_{2}\right),
$$

where $n_{1}$ and $n_{2}$ are the positions of the electron and hole, respectively, and

$$
W_{o}(n)=\frac{e \phi_{s}(n, n)}{2}=\frac{e}{2 C_{e f f}}\left[1-\frac{\cosh [(N-2 n) \lambda]-e^{-N \lambda}}{\sinh (N \lambda)}\right]
$$

is the self-energy of an electron or hole.

Figure 1 shows the results of calculation of the crossover current using Eqs. (25) and (22) in a fixed scenario picture, where the electron and hole enter the array one right after 
the other, and then take turns tunneling towards each other in a symmetric manner. For $N \lambda \gtrsim 15$, the results of this semi-analytical calculation match the Monte Carlo results very closely. (For $N \lambda \lesssim 15$, a difference appears, increasing with $N$.) This means that we can analyze our problem, at least approximately, by examining the energy profile in our fixed scenario. A straightforward analysis of Eq. (25) shows that, for $N \lambda \gg 1$, there is a slow point (a minimum in $\Delta W_{i j}$ ) when both electron and hole are $\sim N / 3$ islands from their respective edge of the array. Here both members of the electron-hole pair are far from the edges and cannot be pushed strongly by the external voltage, yet are not close enough to attract each other strongly, either. At this point, $\Gamma$ scales as $\exp (-N \lambda / 3)$. According to Eq. (22), this leads to a similar dependence of $I_{c}$, at least in the limit $N \lambda \gg 1$.

Some difference between the fixed-scenario calculations and the Monte Carlo simulations can be readily explained by the observation that frequently the tunneling process is somewhat more complicated than the exactly alternating electron and hole motion sequence- see Table 1. Figure 5 shows a typical pattern of the total tunneling rates.

These figures show that sometimes there are three (rather then one) slow points with low $\Gamma$. The first slow point is before any charge enters the array (see labels (a) in Fig. 5). After the first charge enters the array, the external voltage pushes it quickly away from the edge. There is then typically a second slow point when that charge reaches a distance of

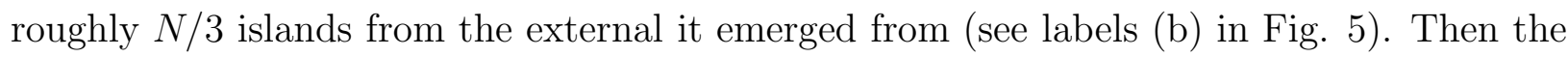
opposite charge enters the array from the other side, also hopping quickly away from the edge. The third slow point usually follows when the opposite charge is roughly $N / 3$ islands away from its external (see labels (c) in Fig. 5). Only the first two points are taken into account in our fixed scenario. Since the second slow point is frequently important, the good agreement between our Monte Carlo and fixed scenario calculations is somewhat puzzling. 


\section{MODEL 2: ARRAYS WITHOUT GROUND PLANE}

Here we examine the case of an array with islands between semi-infinite external electrodes (Fig. 6a), without a ground plane. In the absence of a ground plane, mutual capacitances other than the junction capacitances may become important.

To model the electrostatics of such arrays, we have used two methods. In the first method, we create a geometric model of the island and external electrodes (Fig. 6]b), and use it to calculate the full capacitance matrix for the array numerically using FastCap 1 . Since the electrostatics is rather insensitive to the exact shape of the islands 17 , they may be modeled by cubes of side length $a$. The resulting capacitance matrix (an example is given in Table [I] was used for the Monte Carlo simulation of noise, as described above.

In the second method, we used a simple heuristic approximation for the single-electron soliton potential at distance $m=\left|n-n^{\prime}\right|$ in a long array, found in Ref. 17,

$$
\phi_{s}(m)=\frac{e}{a}\left\{\alpha \lambda \exp (-\kappa \lambda m)+\frac{1}{m}[1-\exp (-\kappa \lambda m)]\right\} \text {, }
$$

with $\alpha \approx \kappa \approx 1$. This formula describes a crossover from an exponential decay at short distance $(\lambda m<1)$ to a Coulomb-law $1 / r$ decay at large distance. The effect of the external electrodes was described by the usual image charge method; in our case, with two electrodes, it involves an infinite series of images. As a result, the full single electron potential, $\phi_{s}^{h}\left(n, n^{\prime}\right)$, may be expressed as the sum of an infinite series over all image charge contributions, and the self energy can be written as

$$
\begin{aligned}
W_{o}^{h}(n) & =\frac{e \phi_{s}^{h}(n, n)}{2} \\
& =\sum_{i=1}^{\infty}\left\{W_{a}(2 N i)-\frac{\left[W_{a}(2 N(i-1)+2 n)+W_{a}(2 N i-2 n)\right]}{2}\right\}
\end{aligned}
$$

On the other hand, the form of the external potential is quite simple,

$$
\phi_{e}^{h}(n) \simeq e V(N-2 n) / 2 N
$$

The energy for an electron-hole pair can then be written just as in Eqn. (25). This energy 
was used for the fixed-scenario calculation of noise, similar to that described in the previous section.

Results from both methods are shown in Fig. 7. The Monte Carlo simulation shows that, unlike in arrays near a ground plane, $I_{c}$ does not follow a universal dependence on $N \lambda$. Rather, the $I_{c}$ values follow a common curve depending mostly on $\lambda$ alone, decreasing weakly with the array length $N$. Even the $\lambda$ dependence is weak, compared to the results for arrays near ground plane: for $N=20$ in the range of $\lambda$ from 0.25 to $1, I_{c}$ drops by a factor of $\sim 3$ in arrays with no ground plane, whereas it drops by more than two decades in arrays with ground plane (Fig. 田).

The fixed scenario results for $I_{c}$ match the Monte Carlo results fairly well in terms of the shape of the $\lambda$ dependence of the curves. For $N=10$, the magnitude of the results are also in fairly close agreement. However, the fixed scenario results exhibit a stronger decline with $N$ than the Monte Carlo results. For $N=60$, the fixed scenario values of $I_{c}$ are between 3 and 8 times smaller than the corresponding Monte Carlo values.

Generally, it is easy to understand why the fixed scenario values for $I_{c}$ fall below the values calculated with Monte Carlo simulation: randomness of jump location, which is ignored in the fixed scenario calculation, can be thought of as an additional source of noise. As the general level of noise in the crossover region increases, so does the crossover current. However, we are still in need of a simple interpretation of the $N$ and $\lambda$ dependence of the shot noise in arrays without ground plane.

\section{MODEL 3: ARRAYS WITH RANDOM BACKGROUND CHARGE}

Returning to arrays near ground plane, with their simple electrostatics, we have explored the behavior of shot noise in the presence of random background charges on the islands, $\vec{q}_{0}$. These charges can represent, for example, the effect of charged impurities in a substrate.

The charge $q_{0, i}$ placed on each island was randomly selected 3 , using a uniform probability distribution between $-e / 2$ and $e / 2$. (Any integer part of the background charge would 
immediately be compensated by trapping one or a few tunneling electrons or holes.)

The $S-I$ curves which stem from the Monte Carlo simulation of such arrays still show the progression from shot noise to suppressed shot noise, but at larger values of $\lambda$ tend to feature strong, irregular peaks (Fig. 3) where curves for arrays without background charge were smooth. Occasionally these peaks in the $S-I$ curve cause it to cross the $1 / \sqrt{N}$ line more than once. In such cases, we arbitrarily define $I_{c}$ as the lowest crossing value. It turns out that the variation in the general location of $I_{c}$ among different instantiations of $\vec{q}_{0}$ is large enough to make the distinctions among different crossing points on a single curve irrelevant.

Figure 8 shows the average value of $I_{c}$ and its standard deviation of over ensembles of 50 different $\vec{q}_{0}$ realizations. Strikingly, for $\lambda \gtrsim 1, I_{c}$ for all values of $N$ appears to fall on the same, almost flat curve. This behavior is in sharp contrast to results for similar arrays without background charge (Fig. 母). This quasi-universal value of $I_{c}$ is $\sim 5 \times 10^{-3} e / R C$. Although there appears to be weak downward trend in $\left\langle I_{c}\right\rangle$ with increasing $\lambda$ for $\lambda \gtrsim 1.5$, this trend is virtually hidden within the relatively large standard deviation.

Let us try to comprehend this result. Simulation shows that in such arrays the typical tunneling process near the crossover is due to several (rather than one) electron-hole pairs moving simultaneously in the array. (Due to this multiplicity, a fixed-scenario calculation of noise would not make sense.) This effect is easy to explain. The electric potential induced by the background charges creates a series of charge traps. So, as the applied voltage is increased, the first several charges to enter the array are trapped, forming a "sandpile" 9 of charge (see Fig. 9 ). The final energy profile in the sandpile is still random, though the maximum possible energy change $\Delta W_{i j}$ is now upwardly bound by $\Delta W_{\max } \approx e^{2} / 2 C_{\text {eff }}$. The remaining disorder is, however, strong enough to overcome the interaction of distant charges, which is exponentially weak in arrays over a ground plane.

This argument also explains why the crossover current is independent of the array length. We are, however, still in need of an analytical theory which would explain the virtual independence of the average crossover on $\lambda$, and also would predict its universal value cited 
above.

\section{CONCLUSION}

We have used both Monte Carlo and fixed scenario techniques to calculate the lowfrequency current noise for three different models of 1D single-electron-tunneling arrays. Within each of the three models, we find a crossover of the spectral density of current fluctuations, $S_{I}(0)$, from the Schottky value $2 e\langle\bar{I}\rangle$, to the "reduced Schottky" value $2 e\langle\bar{I}\rangle / N$, with increasing current. The crossover can be well characterized in terms of the crossover current $I_{c}$, which may be said to mark the onset of quasicontinuous charge transfer. The particular behavior of $I_{c}$ as a function of $N$ and $\lambda$ depends on the interaction of electrons within the array and on the interaction of electrons with the external field.

For arrays near ground plane, with no background charge, the crossover current exhibits a universal behavior that is a function of only the product $N \lambda$, i.e. of the ratio of the array length $N$ to the length $M=\lambda^{-1}$ of single-electron solitons. At $N \lambda>10$, the dependence is exponential: $I_{c} \propto \exp (-N \lambda / 3)$. Our analysis has shown that this behavior is the result

of the exponential decrease of the soliton interaction with the external electrodes and its counterpart in the electron-hole pair.

In arrays without a ground plane, $I_{c}$ is almost independent of $N$ and exhibits a nearlyuniversal weak decrease with $\lambda$. We believe that the substantial difference in results between this model and the previous model is due to the long-range electrostatic interactions, which were screened by the ground plane in the previous model.

Finally, in arrays with random $\vec{q}_{0}$, we have found that the crossover takes place at a nearly universal value of current, $I_{c} \sim 5 \times 10^{-3} e / R C$. The absence of noise dependence on the array length can be readily explained as a result of the random potential created by the background charges, which overwhelms long range order in the arrays. However, the independence of noise on the single-electron soliton length still has to be explained. 


\section{ACKNOWLEDGMENTS}

The authors thank D.V. Averin and A.N. Korotkov for fruitful discussions, and A. Huq for technical assistance.

${ }^{1}$ D. V. Averin and K. K. Likharev, Mesoscopic Phenomena in Solids (Elsevier, Amsterdam, 1991), p. 173.

${ }^{2}$ Single Charge Tunneling, edited by H. Grabert and M. Devoret (Plenum, New York, 1992).

${ }^{3}$ D. V. Averin and K. K. Likharev, J. Low Temp. Phys. 62, 345 (1986).

${ }^{4}$ K. K. Likharev and A. B. Zorin, J. Low Temp. Phys. 59, 347 (1985).

${ }^{5}$ L. S. Kuzmin and D. Haviland, Phys. Rev. Lett. 67, 1161 (1991).

${ }^{6}$ P. Delsing, in Ref. 2, p. 249.

${ }^{7}$ K. A. Matsuoka, 1997. Software available at http://hana.physics.sunysb.edu/mso/.

${ }^{8}$ N. S. Bakhvalov, G. S. Kazacha, K. K. Likharev, and S. I. Serdyukova, Sov. Phys. JETP 68, $581(1989)$.

${ }^{9}$ A. A. Middleton and N. S. Wingreen, Phys. Rev. Lett. 71, 3198 (1993).

10 J. A. Melsen, U. Hanke, H. O. Müller, and K. A. Chao, Phys. Rev. B 55, 10638 (1997).

${ }^{11}$ A. N. Korotkov, unpublished (1996).

12 A. N. Korotkov, Phys. Rev. B 50, 17674 (1994).

${ }^{13}$ W. H. Press, S. A. Teukolsky, W. T. Vetterling, and B. P. Flannery, Numerical Recipes in C (Cambridge Univ. Press, Cambridge, 1992), pp. 278-282.

${ }^{14}$ M. J. M. de Jong and C. W. J. Beenakker, in Mesoscopic Electron Transport, edited by L. L. 
Sohn, L. P. Kouwenhoven, and G. Schön (Kluwer Academic, Dordrecht, 1997).

${ }^{15}$ N. S. Bakhvalov, G. S. Kazacha, K. K. Likharev, and S. I. Serdyukova, Physica B 173, 319 (1991).

${ }^{16}$ K. Nabors, S. Kim, and J. White, IEEE Trans. on Microwave Theory and Techniques 40, 1496 (1992), software available at ftp://rle-vlsi.mit.edu/pub/fastcap/.

${ }^{17}$ K. K. Likharev and K. A. Matsuoka, Appl. Phys. Lett. 67, 3037 (1994). 


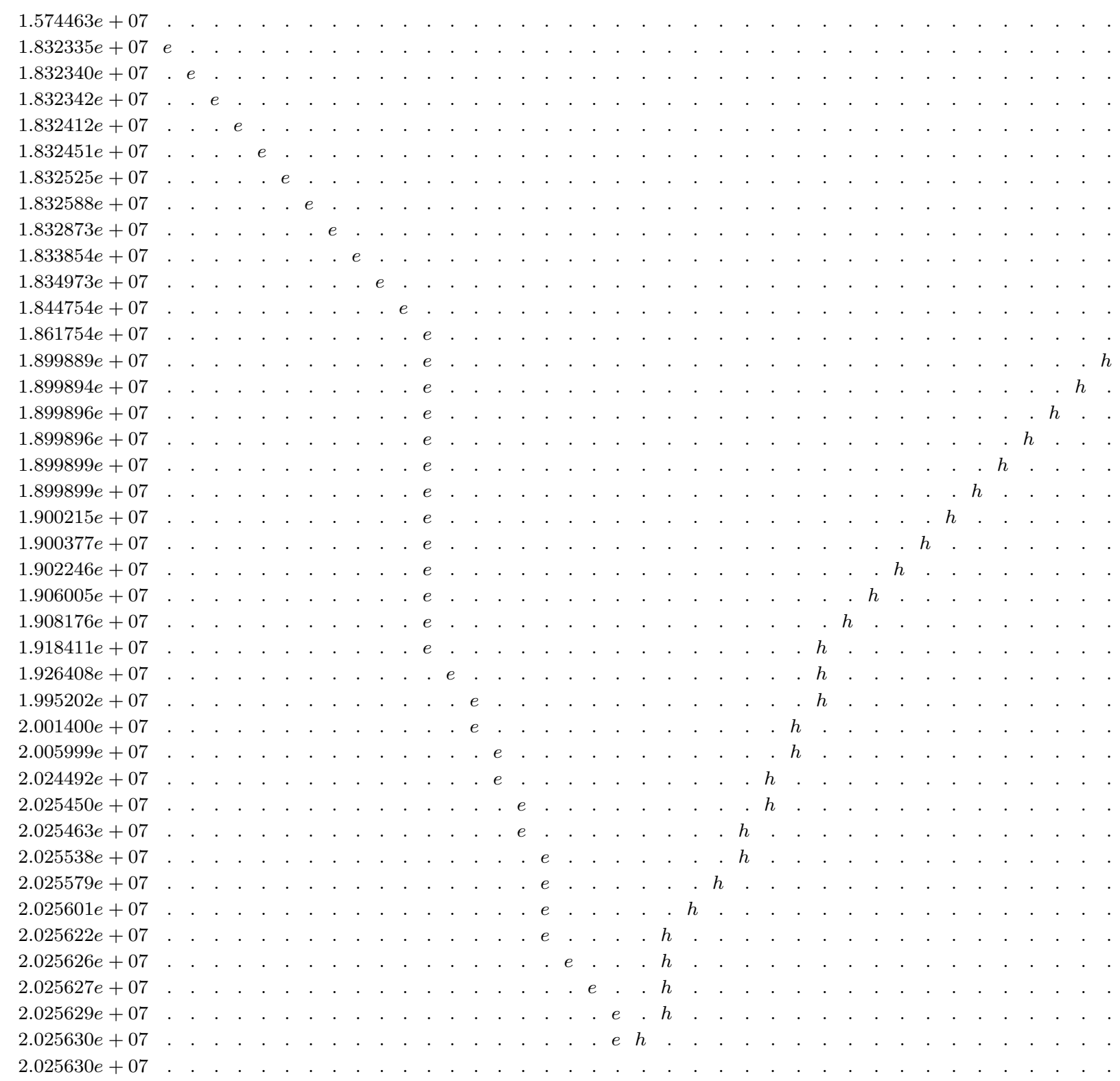

TABLE I. Conduction diagram in a Monte Carlo simulation of an array near a ground plane with no background charge, symmetrically biased near threshold, $N=40, \lambda=0.96$. The first column is time, subsequent columns show the charge on each island. 


\begin{tabular}{|c|c|c|c|c|c|c|c|c|c|c|}
\hline & 3 & 4 & 5 & 6 & 7 & 8 & 9 & 10 & 11 & 12 \\
\hline 1 & 2.99525 & 0.33124 & 0.25056 & 0.20843 & 0.18008 & 0.15821 & 0.13997 & 0.12453 & 0.11055 & 0.09774 \\
\hline 2 & 0.00456 & 0.01429 & 0.02383 & 0.03347 & 0.04319 & 0.05314 & 0.06333 & 0.07441 & 0.08573 & 0.09768 \\
\hline 3 & & & & & & & & & & \\
\hline 4 & 2.56553 & & & & & & & & & \\
\hline 5 & -0.01817 & 2.59339 & & & & & & & & \\
\hline 6 & -0.00665 & -0.02876 & 2.59838 & & & & & & & \\
\hline 7 & -0.00319 & -0.01199 & -0.03172 & 2.60029 & & & & & & \\
\hline 8 & -0.00181 & -0.00632 & -0.01391 & -0.03303 & 2.60122 & & & & & \\
\hline 9 & -0.00115 & -0.00385 & -0.00764 & -0.01485 & -0.03372 & 2.60174 & & & & \\
\hline 10 & -0.00070 & -0.00267 & -0.00473 & -0.00834 & -0.01537 & -0.03412 & 2.60205 & & & \\
\hline 11 & -0.00050 & -0.00172 & -0.00323 & -0.00531 & -0.00874 & -0.01567 & -0.03434 & 2.60223 & & \\
\hline 12 & -0.00037 & -0.00122 & -0.00228 & -0.00357 & -0.00562 & -0.00897 & -0.01584 & -0.03448 & 2.60231 & \\
\hline 13 & -0.00029 & -0.00097 & -0.00163 & -0.00259 & -0.00387 & -0.00576 & -0.00902 & -0.01595 & -0.03452 & 2.60231 \\
\hline 14 & -0.00022 & -0.00077 & -0.00130 & -0.00186 & -0.00270 & -0.00405 & -0.00611 & -0.00906 & -0.01591 & -0.03447 \\
\hline 15 & -0.00017 & -0.00055 & -0.00096 & -0.00142 & -0.00200 & -0.00281 & -0.00407 & -0.00585 & -0.00910 & -0.01585 \\
\hline 16 & -0.00013 & -0.00041 & -0.00073 & -0.00107 & -0.00149 & -0.00203 & -0.00276 & -0.00400 & -0.00580 & -0.00897 \\
\hline 17 & -0.00010 & -0.00032 & -0.00055 & -0.00080 & -0.00110 & -0.00149 & -0.00197 & -0.00276 & -0.00382 & -0.00562 \\
\hline 18 & -0.00009 & -0.00026 & -0.00041 & -0.00057 & -0.00076 & -0.00100 & -0.00137 & -0.00190 & -0.00265 & -0.00365 \\
\hline 19 & -0.00002 & -0.00011 & -0.00025 & -0.00042 & -0.00063 & -0.00088 & -0.00121 & -0.00121 & -0.00154 & -0.00223 \\
\hline 20 & -0.00002 & -0.00008 & -0.00015 & -0.00023 & -0.00033 & -0.00046 & -0.00063 & -0.00069 & -0.00092 & -0.00124 \\
\hline 21 & -0.00001 & -0.00003 & -0.00005 & -0.00007 & -0.00010 & -0.00014 & -0.00019 & -0.00021 & -0.00028 & -0.00037 \\
\hline
\end{tabular}

TABLE II. Partial capacitance matrices generated by FastCap for a cubic array with $N=20$ cubes between large parallel plates. Rows 1 and 2 belong to $\tilde{\boldsymbol{C}}$, rows 3-21 belong to $\boldsymbol{C}$. By convention, all $\tilde{C}_{i j}>0$. All values in $10^{-16} \mathrm{~F}$. The array parameters are $b=1.0, d=0.04$, $\lambda=0.356$. 
FIG. 1. 1D array of tunnel junctions: (a) general schematic, (b) "ground plane" or "tridiagonal model" schematic.

FIG. 2. Convergence of $S_{I}(0) / 2 e I$ calculated by mso as a function of $N_{r}$, the number of current runs in the statistical ensemble, for various values of total transferred charge $Q$. Results for an array near ground plane, $N=20, \lambda=0.5$, at crossover current.

FIG. 3. Parametric plots of $S_{I}(0) / 2 e I$ vs. $I$. Crossover current $I_{c}$ is defined as the current at which $S_{I}(0) / 2 e I=1 / \sqrt{N}$. Circles: array near ground plane. Squares: array without ground plane. Diamonds and triangles: arrays with random background charge. Dotted lines are only guides for the eye.

FIG. 4. Crossover current $I_{c}$ as a function of the $N \lambda$, the ratio of array length to charge soliton length, in arrays near ground plane with no background charge.

FIG. 5. $\Gamma$ vs. time for a Monte Carlo simulation of an array near ground plane with no background charge, symmetrically biased near threshold, $N=20, \lambda=0.55$. Slow points occur before the first charge enters the array (a), and when either charge is $\sim N / 3$ junctions away from the nearest edge $(b, c)$. Dotted line is only a guide for the eye.

FIG. 6. Array, without ground plane, between parallel plate externals. (a) Schematics, (b) geometric model with surfaces divided into panels for geometric capacitance calculation, and (c) closeup of a single island paneling.

FIG. 7. Crossover current vs. inverse soliton length for arrays without ground plane between parallel plate externals.

FIG. 8. Crossover current as a function of inverse soliton length in arrays near ground plane with random background charge. Each point with its error bars represents the average and standard deviation over ensembles of fifty different random background charge distributions. Error bar widths are scaled for readability: narrowest for $N=10$, widest for $N=40$. 
FIG. 9. Typical charge distribution in array with random background charges $\vec{q}_{0} . \quad N=20, \lambda=2$. Filled bars represent $\vec{q}_{0}$, solid lines represent the sandpile at $V=V_{t}$, and dotted lines represent conduction charges in a simple alternating $e-h$ scenario.

FIG. 10. Histograms of the inverse total tunneling rate $1 / \Gamma$ at $I=I_{c}$ for an array near ground plane $(N=40, \lambda=0.995)$ with several different random charge distributions. $r_{q}$ is the seed given to the random number generator for background charges. 


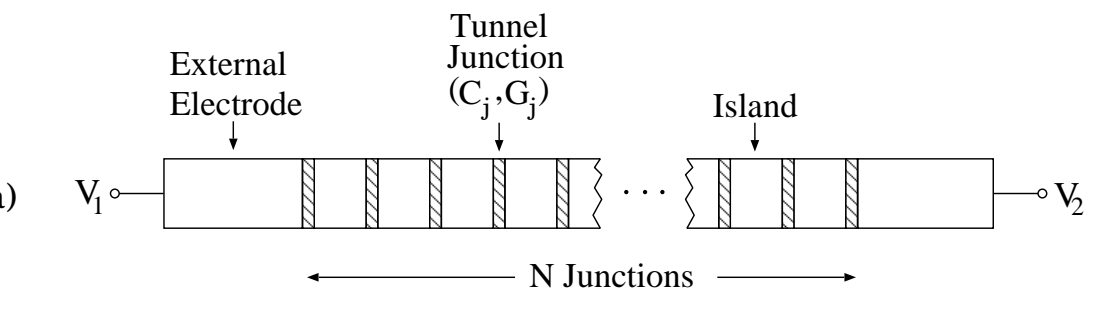

b)

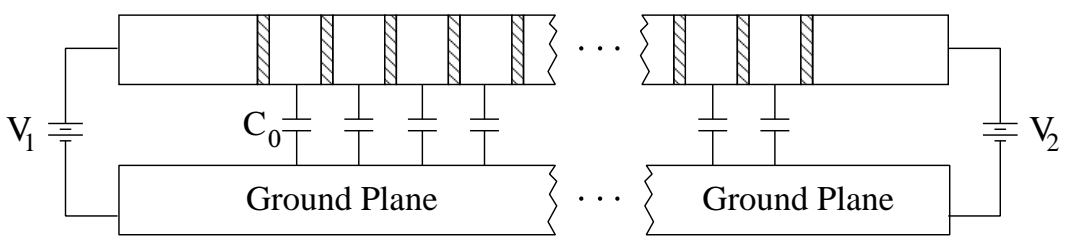

Fig. 1 


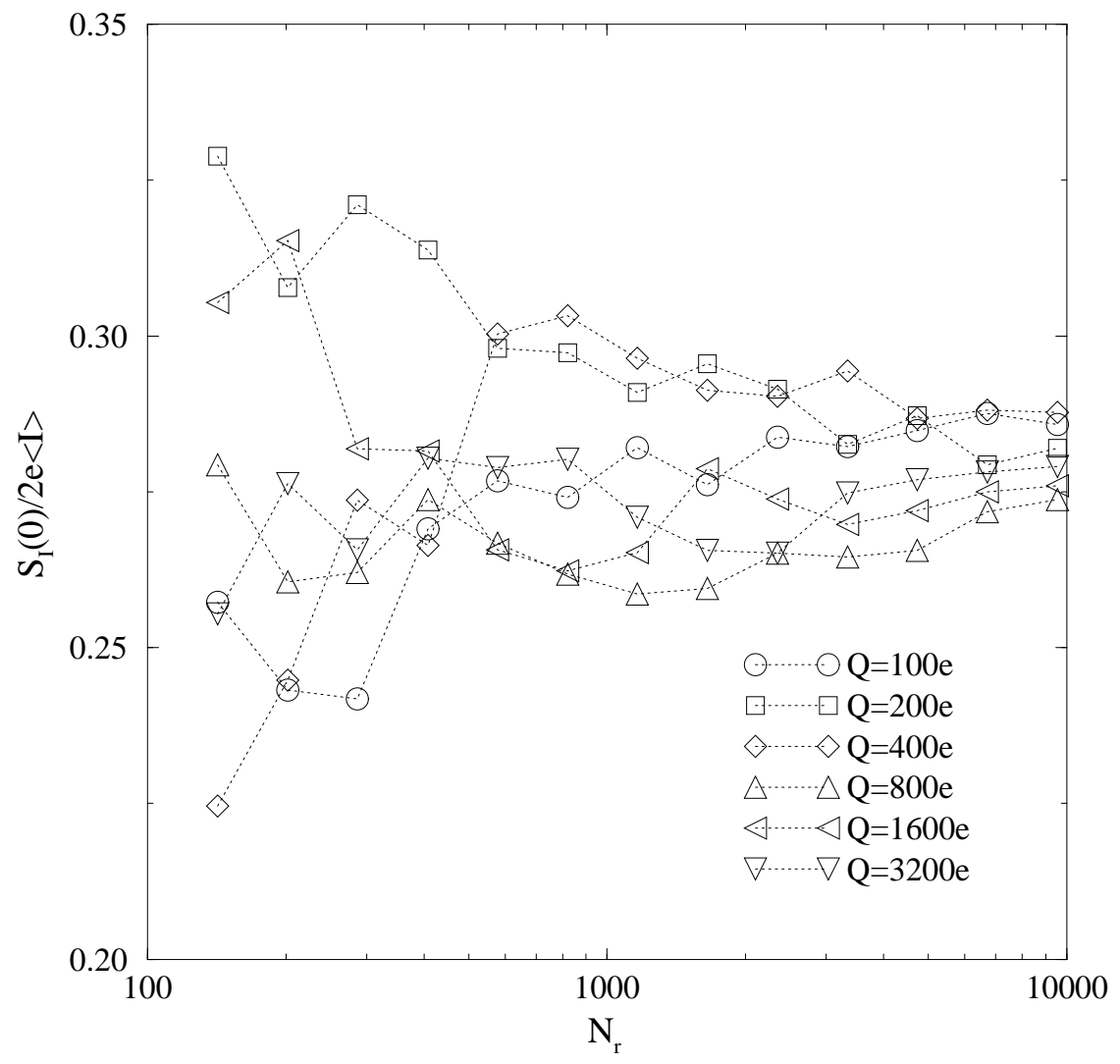

Fig. 2 


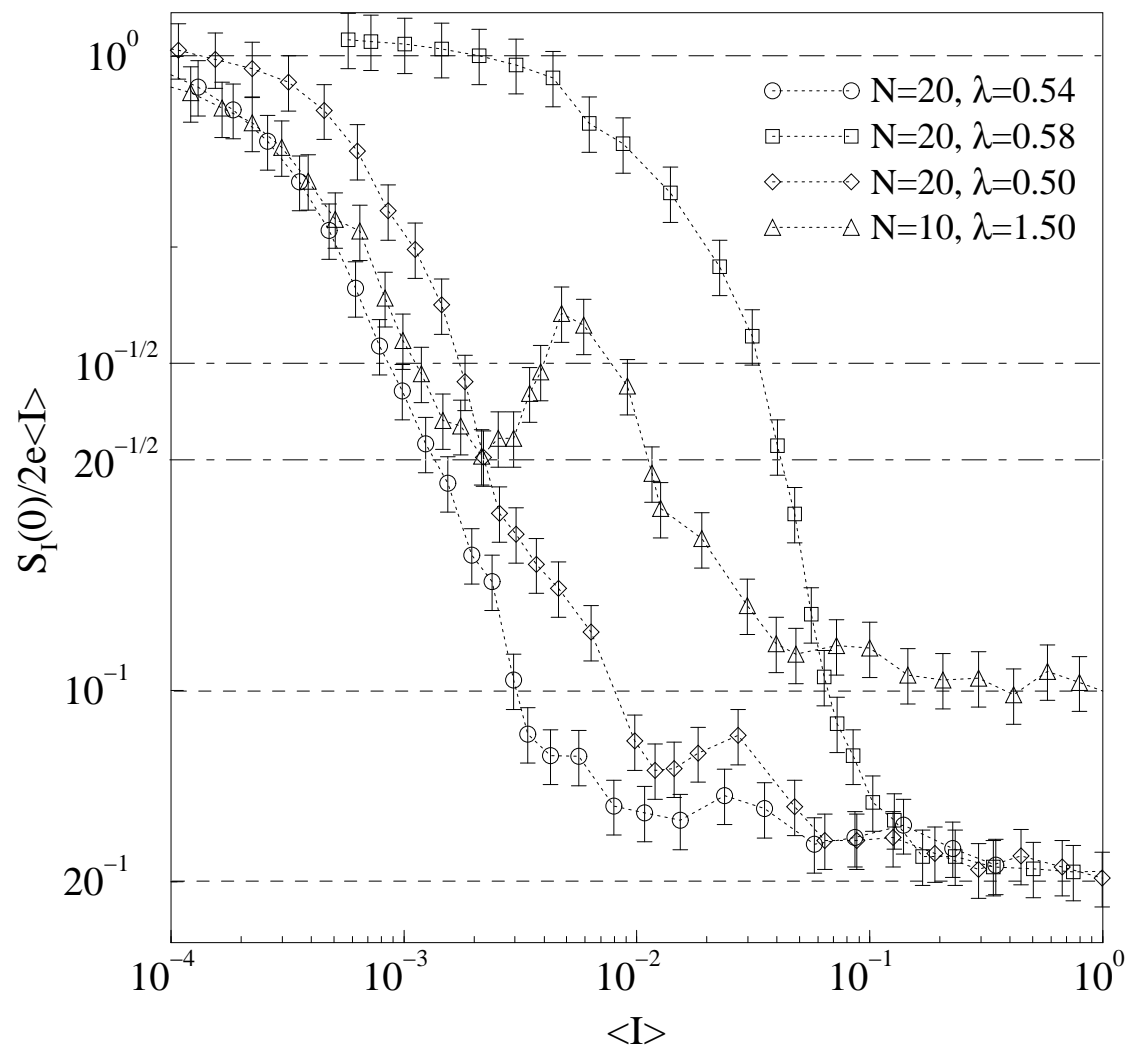

Fig. 3 

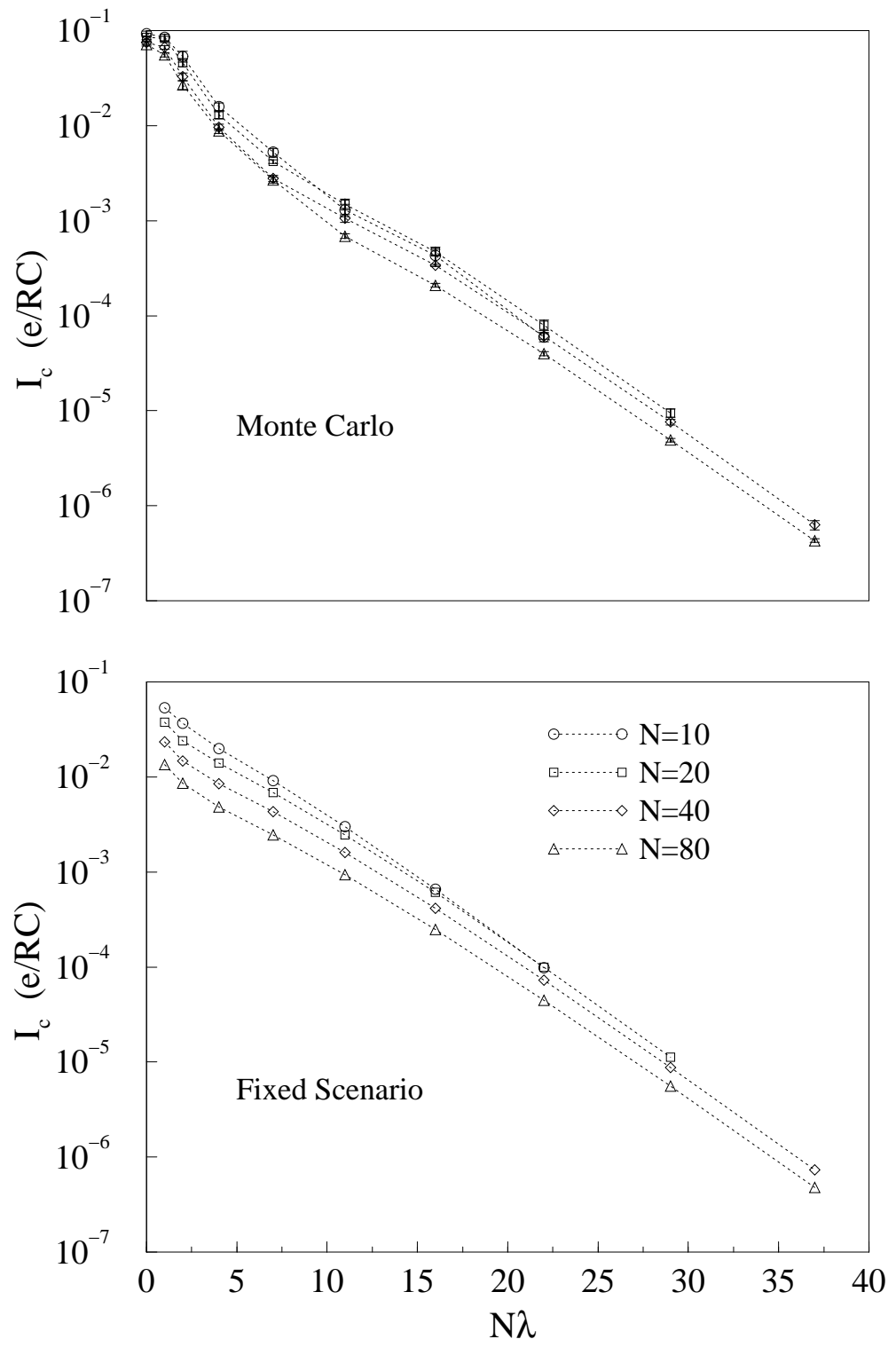

Fig. 4 

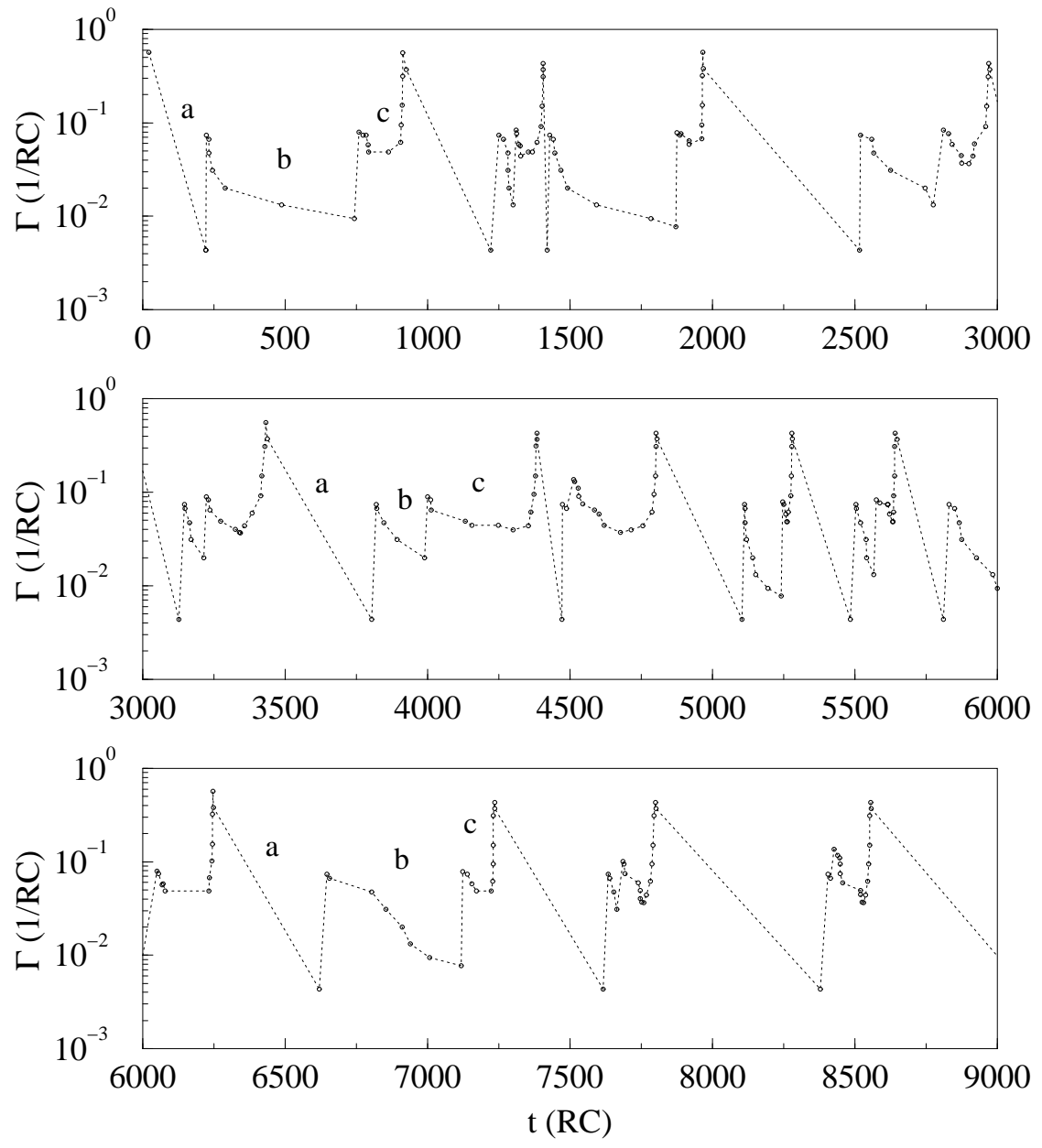

Fig. 5 


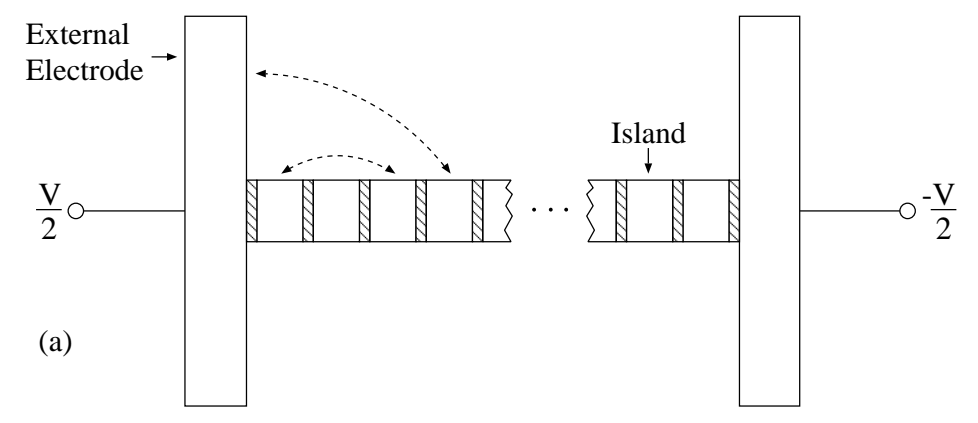

(b)
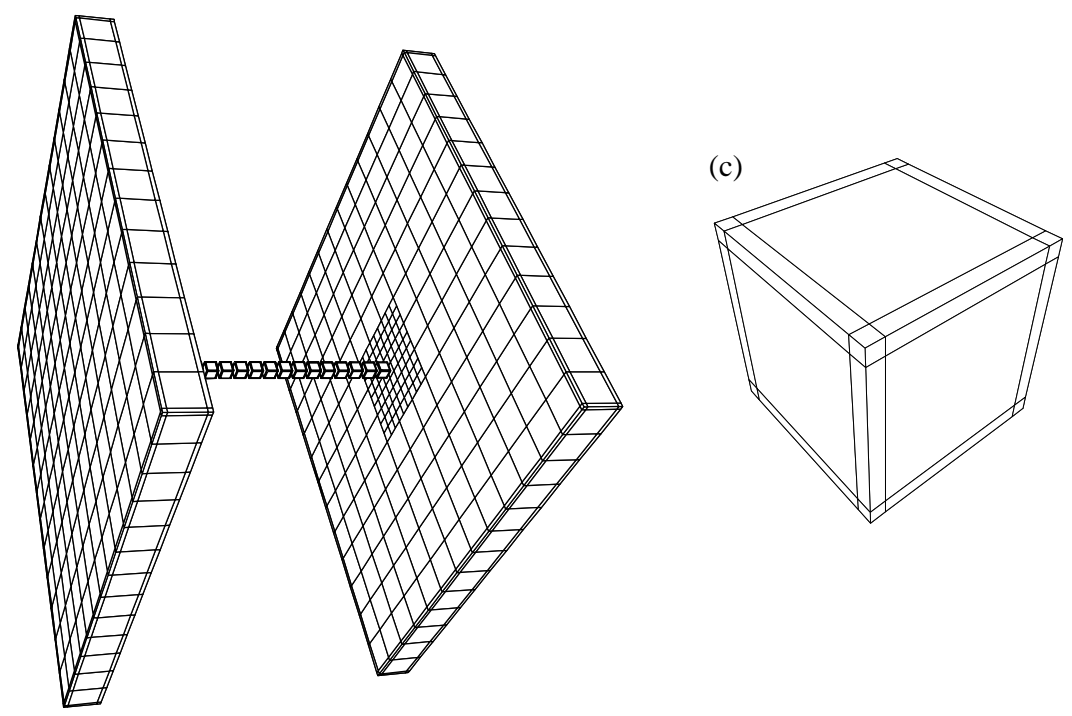

Fig. 6 

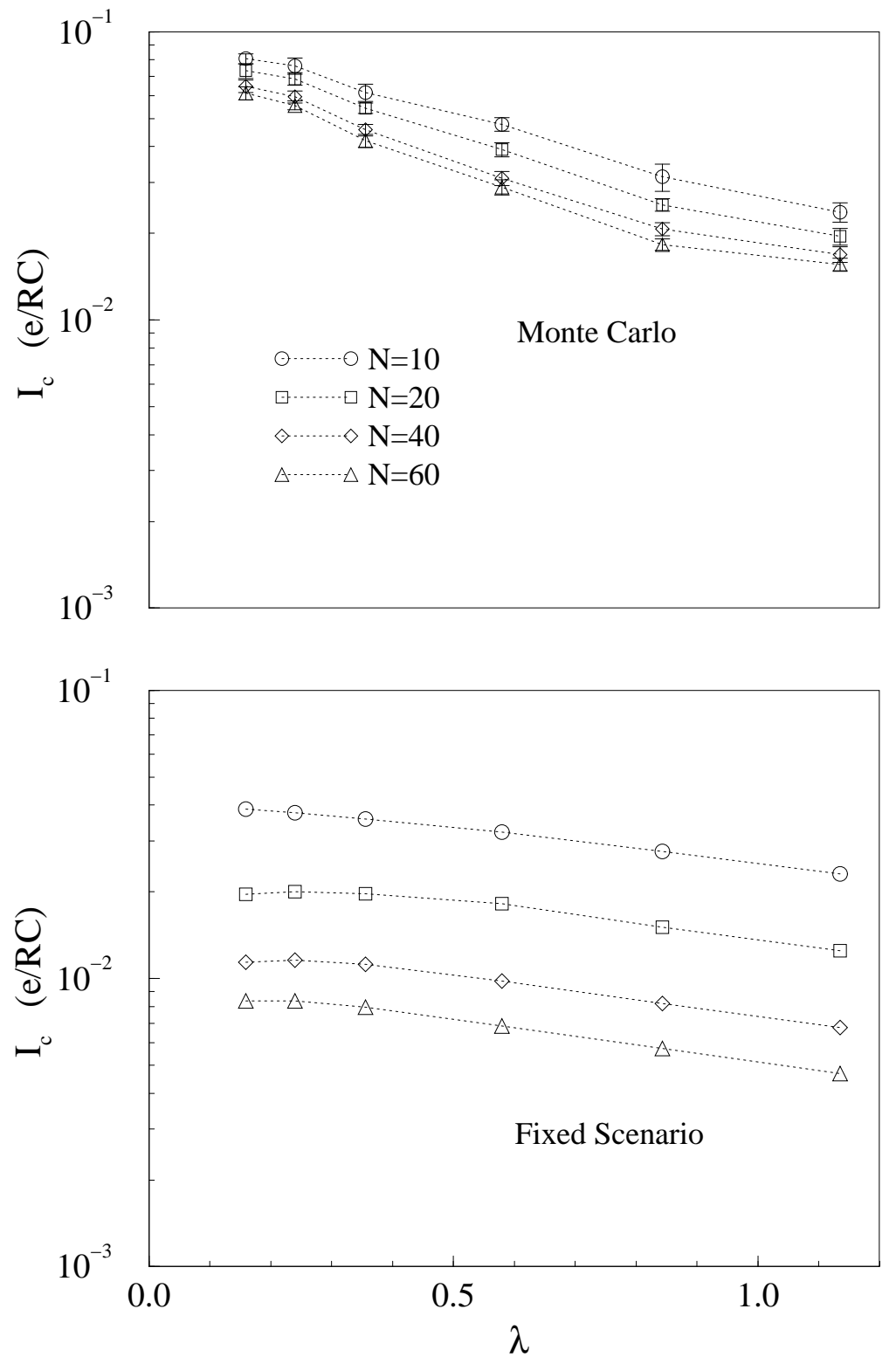

Fig. 7 


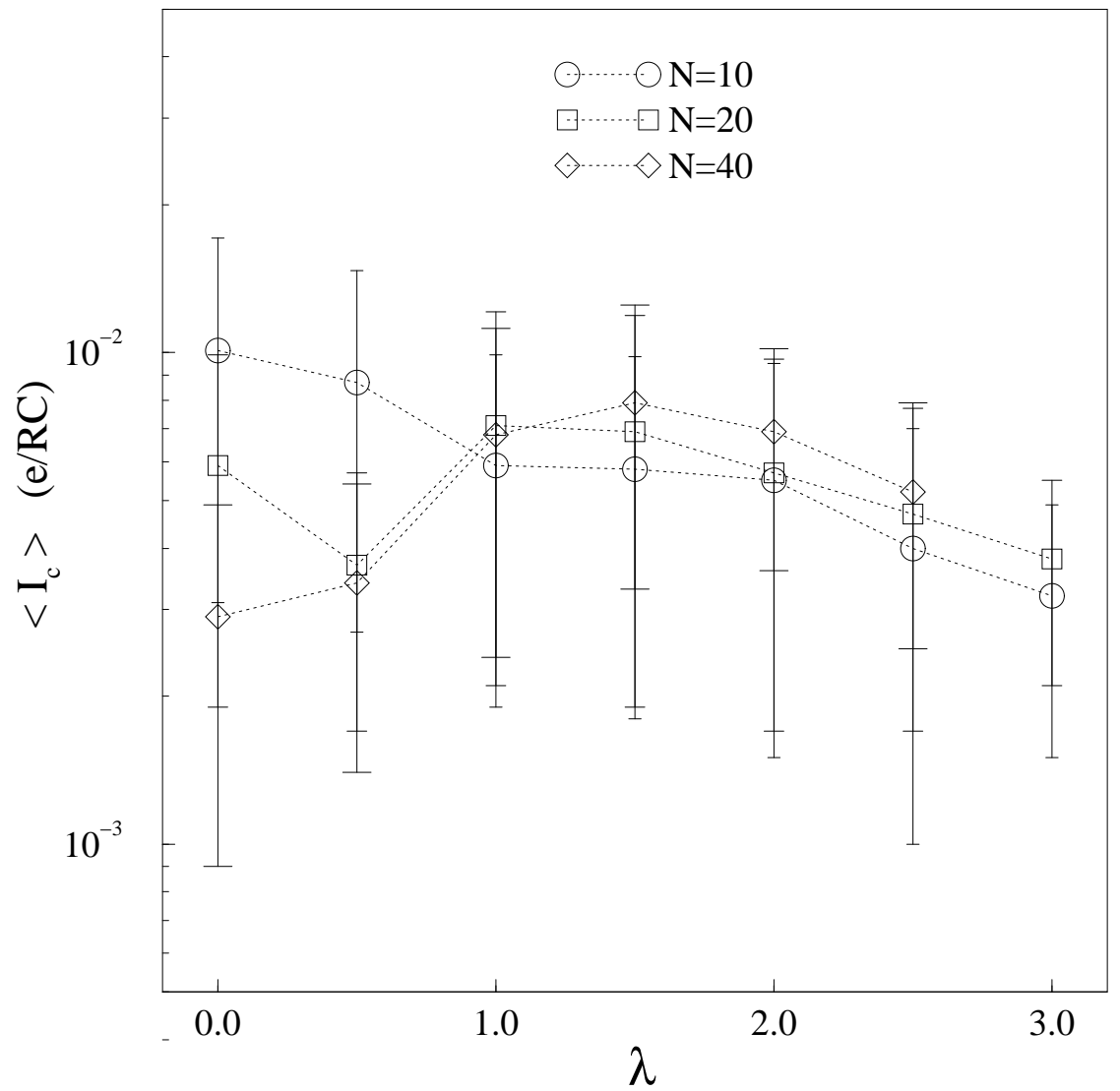

Fig. 8 


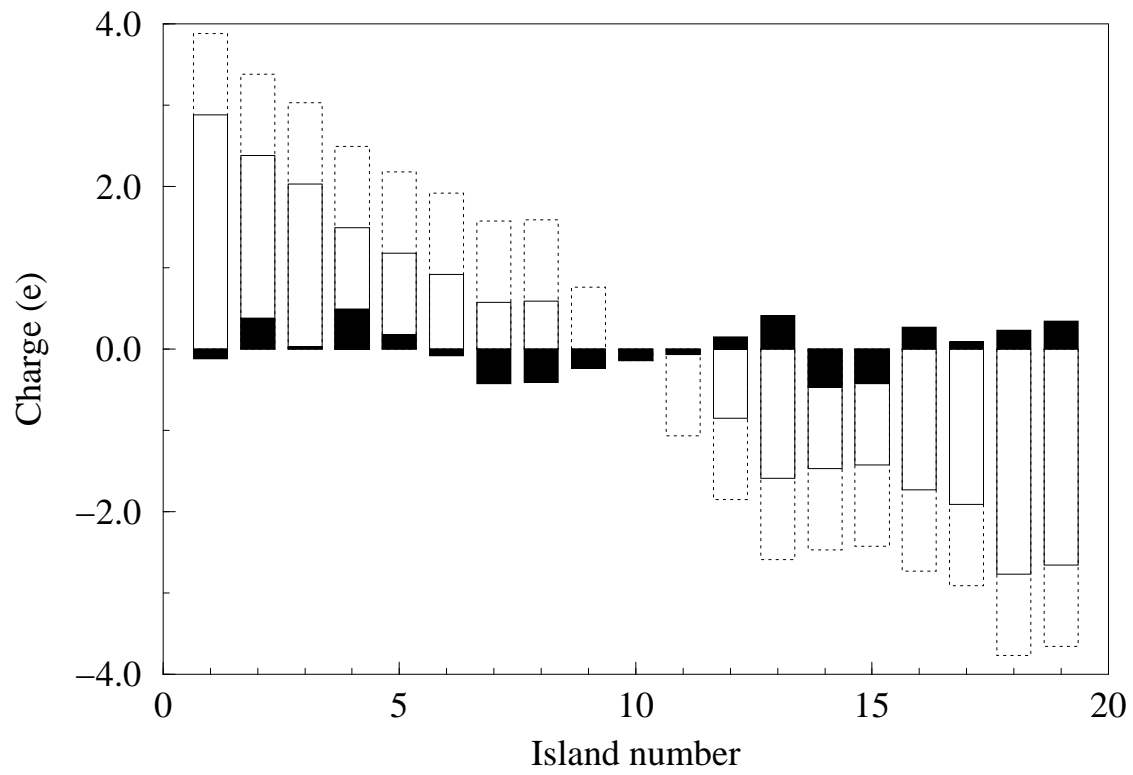

Fig. 9 

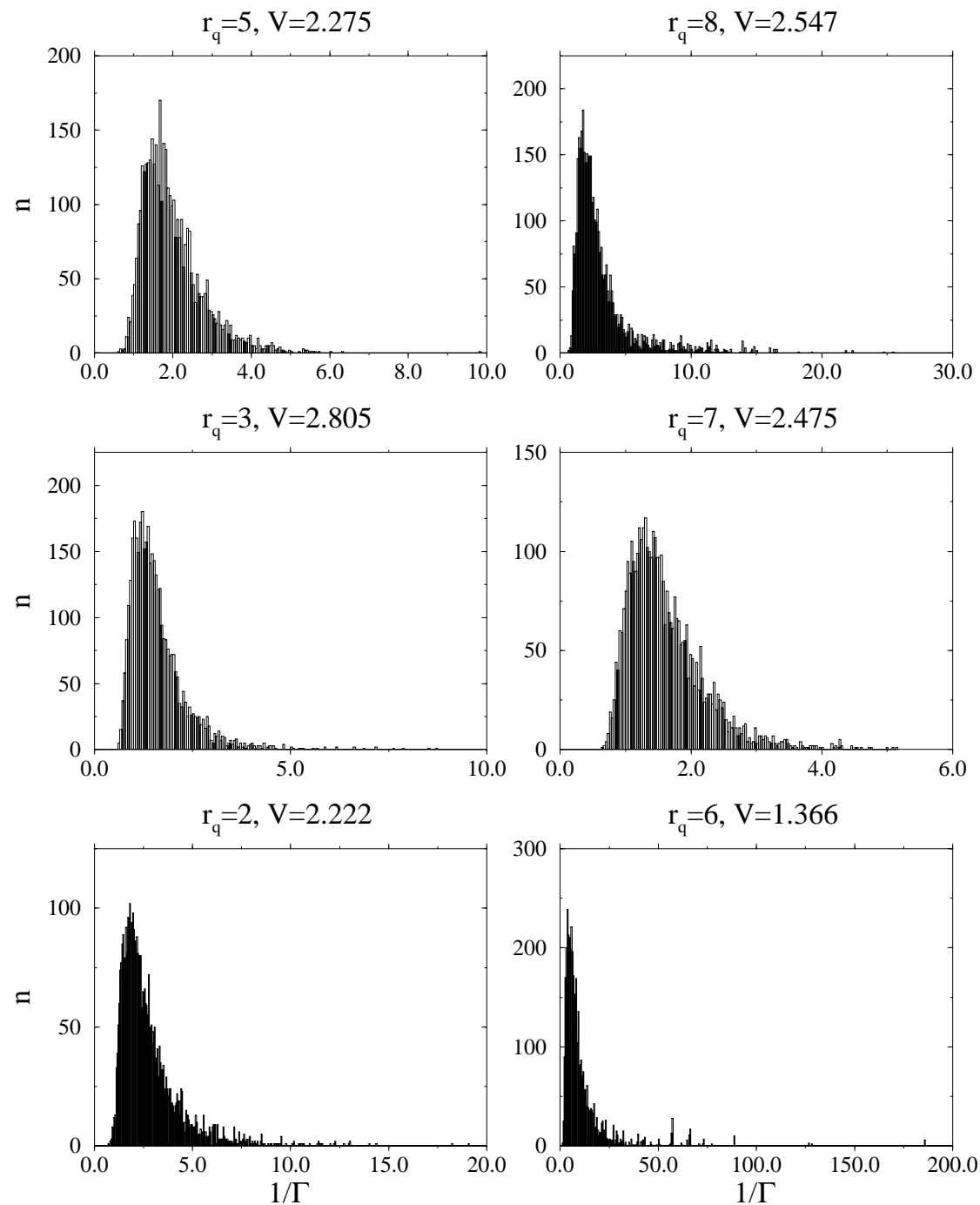

Fig. 10 